( Д.Н. Лаптев ${ }^{1}$, А.О. Емельянов ${ }^{1 *}$, Ю.Г. Самойлова², Е.Б. Храмова ${ }^{3}$, Е.Е. Петряйкина ${ }^{4}$, И.Г. Рыбкина ${ }^{4}$, А.Ю. Филимонова ${ }^{5}$, В.А. Петеркова ${ }^{1}$

'Национальный медицинский исследовательский центр эндокринологии, Москва, Россия

${ }^{2}$ Сибирский государственный медицинский университет, Томск, Россия

${ }^{3}$ Тюменский государственный медицинский университет, Тюмень, Россия

${ }^{4}$ Морозовская детская городская клиническая больница, Москва, Россия

${ }^{5}$ Областная детская клиническая больница имени Н.В. Дмитриевой, Рязань, Россия

ОБОСНОВАНИЕ. Непрерывная подкожная инфузия инсулина (НПИИ) является эффективным методом оптимизации гликемического контроля у детей с сахарным диабетом 1 типа (СД1). Однако использование НПИИ не всегда сопровождается удовлетворительным гликемическим контролем. Одним из методов повышения эффективности проводимого лечения может стать использование телемедицины.

ЦЕЛЬ. Оценить использование удаленной поддержки детей и подростков с СД1 и ее влияние на гликемический контроль, качество жизни и частоту острых осложнений СД1.

МЕтоды. Проведено 24-недельное многоцентровое проспективное открытое контролируемое клиническое исследование. В него были включены 180 детей и подростков, отвечающих следующим критериям: 1) возраст 8-18 лет; 2) СД1 не менее 1 года; 3) помповая инсулинотерапия Medtronic Paradigm (Medtronic MiniMed, США) не менее 6 мес; 4) самоконтроль гликемии не менее 4 раз в сутки и замена инфузионной системы инсулиновой помпы не реже 1 раза

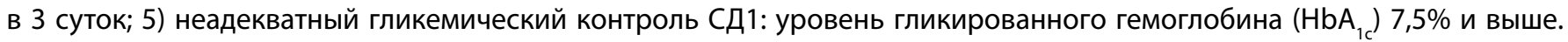
Пациенты были распределены в группу дистанционного консультирования (ДК; $\mathrm{n=100)}$ или традиционного контро-

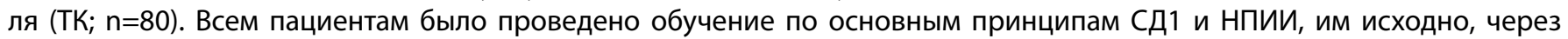
12 и 24 недели проводились измерение $\mathrm{HbA}_{1 c^{\prime}}$ регистрация и анализ показателей гликемии и суточных доз инсулина, оценка и коррекция проводимого лечения. Пациенты или их родители в группе ДК не реже одного раза в 2 недели в домашних условиях отправляли данные помпы через Интернет в центр помповой инсулинотерапии, а в ответ получали рекомендации по лечению.

РЕзУЛЬтАТЫ. Во всех центрах в исследование были включены 180 детей в возрасте 8-18 лет. Пациенты в обеих группах не различались по возрасту, полу, длительности СД1 и НПИИ, а также уровню НbА онных консультаций по всем исследовательским центрам составило 949. Снижение уровня НbА ния относительно исходного было статистически значимо больше в группе ДК: 1,17\% по сравнению с 0,59\% в группе TK ( $<<0,05)$. Доля пациентов, которые достигли целевого уровня $\mathrm{HbA}_{1 c}(<7,5 \%)$, была статистически значимо выше в группе ДК (32\%) по сравнению с группой ТК (12,5\%, p<0,05). За время исследования частота эпизодов диабетического кетоацидоза и тяжелой гипогликемии в группе ДК статистически значимо была ниже.

ЗАКЛЮчЕНИЕ. У детей с СД1 дистанционная поддержка привела к значимому улучшению гликемического контроля ( $\mathrm{HbA}_{1 c^{\prime}}$ вариабельность гликемии, частота гипогликемии). Накопление доказательств эффективности и безопасности телемедицины при СД должно способствовать внедрению данного подхода в практическое здравоохранение.

КЛЮЧЕВЫЕ СЛОВА: СД 1 типа у детей и подростков; инсулиновые помпы; помповая инсулинотерапия; непрерывная подкожная инфузия инсулина; НПИИ; телемедицина.

\title{
REMOTE MONITORING AND TREATMENT OF CHILDREN AND ADOLESCENTS WITH TYPE 1 DIABETES
}

(c) Dmitry N. Laptev ${ }^{1}$, Andrey O. Emelyanov ${ }^{1 *}$, Yulia G. Samoylova², Elena B. Khramova ${ }^{3}$, Elena E. Petriaikina ${ }^{4}$, Irina G. Rybkina ${ }^{4}$, Alla Yu. Filimonova ${ }^{5}$, Valentina A. Peterkova'

'Endocrinology Research Centre, Moscow, Russia

${ }^{2}$ Siberian State Medical University, Tomsk, Russia

${ }^{3}$ Tyumenian State Medical University, Tyumen, Russia

${ }^{4}$ Morozov Children City Clinical Hospital, Moscow, Russia

${ }^{5}$ Regional Children Clinical Hospital named after N.V. Dmitrieva, Ryazan, Russia 
RATIONALE: Continuous subcutaneous insulin infusion (CSII) is an effective method for optimizing glycemic control in children with type 1 diabetes mellitus (DM1). However, the use of CSII does not always result in adequate glycemic control. Telehealth can be applied as one of the methods to improve the effectiveness of treatment.

AIMS: To evaluate the use of remote medical support of children and adolescents with DM1 and its influence on glycemic control, quality of life, and incidence of acute complications of DM1.

MATERIALS AND METHODS: We conducted a 24-week multi-institutional prospective open-label controlled clinical trial. 180 children and adolescents were included in this study and divided into the following categories: 1) age 8-18 years; 2) DM1 at least 1 year; 3) pump insulin therapy Medtronic Paradigm (Medtronic MiniMed, USA) at least 6 months; 4) self-monitoring of glycemia at least 4 times a day and replacement of the insulin pump infusion system at least once every 3 days; 5 ) inadequate glycemic control of DM1: the level of glycated hemoglobin $\left(\mathrm{HbA}_{1 c}\right) 7.5 \%$ or higher. Patients were assigned to a remote consultation group ( $R C ; n=100)$ or a traditional control group (TC; $n=80)$. All patients were trained on the basic principles of DM1 and CSII, and we measured initial HbA1c, then after 12 and 24 weeks, also registered and analyzed glycemic indicators and daily doses of insulin, evaluated and corrected the treatment. Patients or their parents in the RC group sent pump data via the Internet to the pump insulin therapy center at least once every 2 weeks at home and received treatment recommendations in response.

RESULTS: The total number of patients included in the study in all institutions was 180 children at 8-18 years. Patients in both groups did not differ in age, gender, duration of DM1 and CSII, and $\mathrm{HbA}_{1 c}$ level. The total amount of remote consultations for all institutions was 949 . The decrease in the level of $\mathrm{HbA}_{1 \mathrm{c}}$ by the end of the study against the initial one was statistically significantly greater in the RC group: $1.17 \%$ compared to $0.59 \%$ in the TC group ( $p<0.05)$. The proportion of patients who reached the target level of $\mathrm{HbA}_{1 \mathrm{c}}(<7.5 \%)$ was significantly higher in the RC group (32\%) compared to the TC group $(12.5 \%, p<0.05)$. During the study, the incidence of DKA and severe hypoglycemia in the RC group was statistically significantly lower.

CONCLUSIONS: Remote monitoring in children with DM1 resulted in significant improvements in glycemic control (HbA ${ }_{1 c^{\prime}}$ glycemic variability, and hypoglycemic frequency). The accumulation of evidence on the effectiveness and safety of telehealth in DM should contribute to implementing this approach in practical health care.

KEYWORDS: Diabetes Mellitus Type 1; blood glucose; glycemic; glucose monitoring; Insulin infusion system; telemedicine, remote consultation.

\section{ОБОСНОВАНИЕ}

Непрерывная подкожная инфузия инсулина (НПИИ), или помповая инсулинотерапия, является эффективным методом улучшения и поддержания гликемического контроля у детей с сахарным диабетом 1 типа (СД1) [1-7]. Однако использование НПИИ требует от специалиста-эндокринолога соответствующей квалификации и достаточного опыта, непосредственного участия и контакта врача с пациентом и не всегда сопровождается удовлетворительным гликемическим контролем. Так, по данным Федерального регистра сахарного диабета, целевых уровней $\mathrm{HbA}_{1 c}<7,5 \%$ удается достигнуть лишь у $32 \%$ детей и 25\% подростков [8]. Неудовлетворительная компенсация СД1 может быть связана с рядом причин, в том числе с доступностью квалифицированной медицинской помощи (отсутствием детских эндокринологов по месту жительства, отсутствием навыков и опыта работы с инсулиновыми помпами и др.). Учитывая географические и демографические особенности Российской Федерации, одним из методов повышения эффективности проводимого лечения может стать использование телемедицины.

При СД1 использование телемедицины является весьма актуальным из-за терапевтических особенностей этого заболевания. Сахарный диабет - это хроническое заболевание, требующее наблюдения пациента специалистом-эндокринологом. Пациенты с СД1 должны постоянно контролировать показатели гликемии, а врач - вносить изменения в проводимую схему инсулинотерапии в соответствии с этими данными. Отсутствие соответствующей коррекции проводимой терапии, недостаточный самоконтроль, в том числе в результате дефицита обучения, могут привести к неудовлетворительной компенсации СД1. Это, в свою очередь, способствует развитию и прогрессированию острых и хронических осложнений, что ухудшает качество жизни и дальнейший прогноз, а также значительно увеличивает расходы на лечение диабета.

По данным зарубежных исследований, использование телемедицины у детей с СД1 способствует снижению уровня $\mathrm{HbA}_{1 c^{\prime}}$ улучшению качества жизни, снижению экстренных обращений и госпитализаций по поводу диабета [9]. Кроме того, использование телемедицины в целом может позволить сократить затраты, связанные с перемещением пациента, предоставить дополнительные медицинские услуги и равномерно распределить нагрузку на медицинский персонал.

\section{ЦЕЛЬ}

Оценить возможность удаленной поддержки детей и подростков с СД1 и ее влияние на гликемический контроль, качество жизни и частоту острых осложнений СД1.

\section{МЕТОДЫ}

Проект реализован на базе пяти клинических центров Российской Федерации: 
- ФГБУ «Национальный медицинский исследовательский центр эндокринологии» Минздрава России;

- ФГБОУ ВО «Сибирский государственный медицинский университет» Минздрава России;

- ГБОУ ВПО «Тюменский государственный медицинский университет» Минздрава России;

- ГБУЗ «Морозовская детская городская клиническая больница» Департамента здравоохранения города Москвы;

- ГБУ РО «Областная детская клиническая больница имени Н.В. Дмитриевой».

\section{Дизайн исследования}

Проведено 24-недельное многоцентровое проспективное открытое контролируемое клиническое исследование. В пяти исследовательских центрах Российской Федерации в исследование были включены дети и подростки, отвечающие следующим основным критериям: 1) возраст $\geq 8$ и <18 лет; 2) СД1 длительностью не менее 1 года; 3) инсулинотерапия путем НПИИ помпой Medtronic Paradigm (Medtronic MiniMed, США) длительностью не менее 6 мес; 4) по данным отчетов с инсулиновой помпы за последние 3 мес: самоконтроль гликемии в среднем не менее 4 раз в сутки и замена инфузионной системы инсулиновой помпы не реже 1 раза в 3 суток; 5) неадекватный гликемический контроль СД1: уровень $\mathrm{HbA}_{1 с}$ 7,5\% и выше; 6) наличие дома персонального компьютера с выходом в сеть Интернет; 7) подписанное информированное согласие.

Основные критерии исключения были следующими: 1) СД не 1 типа; 2) инсулинотерапия путем множественных инъекций инсулина (МИИ) или помпой, отличной от Medtronic Paradigm; 3) клинически значимые, острые заболевания сердечно-сосудистой, нервной, мочеполовой систем, желудочного-кишечного тракта и заболевания крови; 4) нарушение протокола исследования: нерегулярный самоконтроль глюкозы крови менее 4 раз в сутки, нерегулярная замена инфузионной системы инсулиновой помпы реже 1 раза в 3 суток, неявка на визиты, отсутствие возможности каждые 2 недели отправлять данные с инсулиновой помпы (для группы дистанционного консультирования); 5) отказ от участия в исследовании.

Процедуры исследования и регистрация показателей

Пациенты, включенные в исследование, на первом визите были распределены в группу дистанционного консультирования (ДК; $n=100$ ) или традиционного контроля (TK; $\mathrm{n}=80$ ). Для исключения влияния уровня знаний и навыков на конечный результат всем пациентам, включенным в исследование, на первом визите было проведено структурированное обучение по основным принципам СД1 и НПИИ.

Всем пациентам исходно, через 12 и 24 недели после включения в исследование на очных визитах проводились стандартный осмотр и антропометрия, осуществлялись исследование уровня $\mathrm{HbA}_{1 c}$ методом жидкостной хроматографии на анализаторе DS5 Glycomat (DrewScientific, Нидерланды), или методом реакции агглютинации моноклональных антител на анализатоpe DCA Vantage Analyzer (Siemens, Германия), или фер- ментативным методом на анализаторе BS-400 (Mindray, Китай), регистрация и анализ показателей гликемии и суточных доз инсулина, оценка и коррекция проводимого лечения, давались рекомендации по самоконтролю и наблюдению. Также исходно и в конце исследования проводилась оценка качества жизни детей и родителей.

Регистрация и анализ показателей гликемии (средний уровень гликемии, вариабельность гликемии (SD), измерений глюкозы крови в диапазоне $<4$ и >10 ммоль/л, гликемический 8-точечный профиль) и среднесуточных доз инсулина проводились с использованием программного обеспечения (ПО) CareLink Professional (Medtronic MiniMed, США) за предшествующий дню визита 2-недельный период. Данное ПО позволяет сформировать отчеты, содержащие информацию об основных показателях гликемии, проводимой инсулинотерапии, частоте самоконтроля, замене инфузионного набора и другие данные за выбранный период.

Всем пациентам был предоставлен глюкометр Contour TS (Bayer, Швейцария) и тест-полоски к нему на все время исследования. Пациентам в группе ДК также было предоставлено техническое обеспечение для передачи данных с инсулиновой помпы на персональный компьютер CareLink USB (Medtronic MiniMed, (ША).

\section{Дистанционное консультирование}

Пациенты или их родители, распределенные в группу ДК, каждые 14 дней (с интервалом \pm 3 дня) между очными визитами в домашних условиях должны были отправлять данные, содержащиеся в памяти инсулиновой помпы, специалисту в исследовательский центр, а в ответ получали от него рекомендации по лечению и самоконтролю. Передача данных осуществлялась через сеть Интернет с использованием ПО CareLink Personal (Medtronic MiniMed, США) и технического обеспечения CareLink USB (Medtronic MiniMed, США). Интерпретация и анализ полученных данных во всех исследовательских центрах производились с использованием ПО CareLink Professional (Medtronic MiniMed, США). На основании полученных отчетов специалист исследовательского центра принимал решение о необходимости коррекции проводимой терапии или давал рекомендации по самоконтролю. В зависимости от объема или необходимости получения дополнительной информации от пациента специалист передавал свои рекомендации посредством электронной почты или связывался с пациентом посредством телефонного или видеозвонка через Skype (Skype Communications SARL, Люксембург). По рекомендации врача могли проводиться дополнительные дистанционные консультации без ограничений по частоте.

Оценка качества жизни детей и родителей

Оценка качества жизни детей и родителей проводилась с использованием опросников PedsQL ${ }^{\mathrm{TM}} 3.0$ Diabetes Module. Опросник состоит из 28 элементов (ситуаций), составляющих 5 модулей: Диабет, Лечение I, Лечение II, Беспокойство, Общение. В опроснике детям или родителям предлагается оценить, насколько 
затруднительными для детей были различные ситуации в течение последнего месяца. Каждый элемент может быть оценен по 5-балльной шкале от 0 (никогда) до 4 (почти всегда). Для подсчета полученных результатов баллы по каждому элементу трансформируются в обратном порядке по шкале от 0 до 100 следующим образом: $0=100,1=75,2=50,3=25,4=0$. Затем рассчитывается средний балл по всем элементам в целом и по каждому разделу отдельно. Чем выше балл, тем лучше качество жизни пациента или родителя.

\section{Основной исход исследования}

Изменение $\mathrm{HbA}_{1 c}$ к концу исследования по сравнению с исходным уровнем и доля пациентов, достигших $\mathrm{HbA}_{1 c}$ менее 7,5\%, к концу исследования между группами ДК и ТК.

Дополнительные исходы исследования:

1) изменение к концу исследования по сравнению с исходным уровнем следующих показателей: средний уровень гликемии, вариабельность гликемии (SD), частота измерений уровня глюкозы крови в диапазонах <4 и >10 ммоль/л, показатели качества жизни, показатели приверженности к контролю СД1 (частота определения гликемии, частота «ручных» болюсов, частота использования калькулятора болюса, частота замены инфузионной системы между группами ДК и ТК); 2) частота эпизодов диабетического кетоацидоза (ДКА), отвечающих следующим критериям: гипергликемия (уровень глюкозы в крови >11 ммоль/л), $\mathrm{pH}<7,3$ в венозной крови или бикарбонат сыворотки <15 ммоль /л, кетонемия (бета-гидроксибутират в крови $\geq 3$ ммоль/л) или умеренная/значительная кетонурия $(\geq 2+)$; 3$)$ частота эпизодов тяжелой гипогликемии, отвечающих следующим критериям: событие с тяжелыми когнитивными нарушениями (включая кому и судороги), требующее помощи другого человека для активного введения углеводов, глюкагона или других корректирующих действий; 4) частота обращений за экстренной и неотложной медицинской помощью за время исследования.

\section{Этическая экспертиза}

Протокол исследования одобрен локальным Комитетом по этике. До включения в исследование законные представители пациентов подписали информированное согласие на участие в нем.

\section{Статистический анализ}

Статистическая обработка полученных результатов была произведена с использованием статистического пакета STATISTICA (StatSoft, США). При нормальном распределении количественного признака данные представлены в виде среднего значения и его стандартного отклонения - M (SD) или в виде среднего значения и его стандартной ошибки - M \pm SEM. Различие между количественными признаками оценивалось с помощью метода Манна-Уитни.

Различие между качественными признаками оценивалось с помощью двустороннего точного критерия Фишера. Различие между частотой острых осложнений (ДКА, тяжелая гипогликемия) оценивалось с помощью двухстороннего Z-критерия. Взаимосвязь между двумя показателями оценивалась с использованием корреляционного анализа методом Спирмена. Для всех статистических методов значение $p<0,05$ считалось статистически значимым.

\section{РЕЗУЛЬТАТЫ}

Всего во всех центрах в исследование были включены 180 детей в возрасте от 8 до 18 лет. Исходная характеристика пациентов в группах ДК и ТК представлена в табл. 1. Пациенты в обеих группах не различались по возрасту, полу, длительности СД1 и НПИИ, а также уровню $\mathrm{HbA}_{1 c}$. Общее количество дистанционных консультаций по всем клиническим центрам составило 949 (из них 933 плановые и 16 дополнительных), со средней продолжитель-

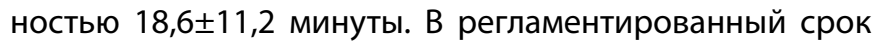
не было проведено по разным причинам (технические проблемы, отклонение пациента/родителя от протокола) 67 плановых дистанционных консультаций.

Таблица 1. Исходная характеристика пациентов по группам. Данные представлены в виде M (SD), н/3 - статистически не значимые различия.

\begin{tabular}{|c|c|c|c|}
\hline & $\begin{array}{c}\text { Группа TK } \\
n=80\end{array}$ & $\begin{array}{c}\text { Группа ДК } \\
n=100\end{array}$ & $\mathbf{P}$ \\
\hline Возраст, годы & $13,1(2,6)$ & $12,6(2,6)$ & $\mathrm{H} / 3$ \\
\hline $\% / M$ & $44 / 36$ & $51 / 49$ & $\mathrm{H} / 3$ \\
\hline Длительность СД1, годы & $5,7(3,2)$ & $4,8(3,1)$ & $\mathrm{H} / 3$ \\
\hline Длительность НПИИ, годы & $3(2,1)$ & $3,8(2,6)$ & $\mathrm{H} / 3$ \\
\hline $\mathrm{HbA}_{1 c^{\prime}} \%$ & $9,4(1,4)$ & $9,1(1,2)$ & $\mathrm{H} / 3$ \\
\hline Poct, $\mathrm{CM}$ & $155,2(14,6)$ & $154,4(15,7)$ & $\mathrm{H} / 3$ \\
\hline Вес, кг & $48,6(15,9)$ & $46,5(15,5)$ & $\mathrm{H} / 3$ \\
\hline
\end{tabular}


10,0

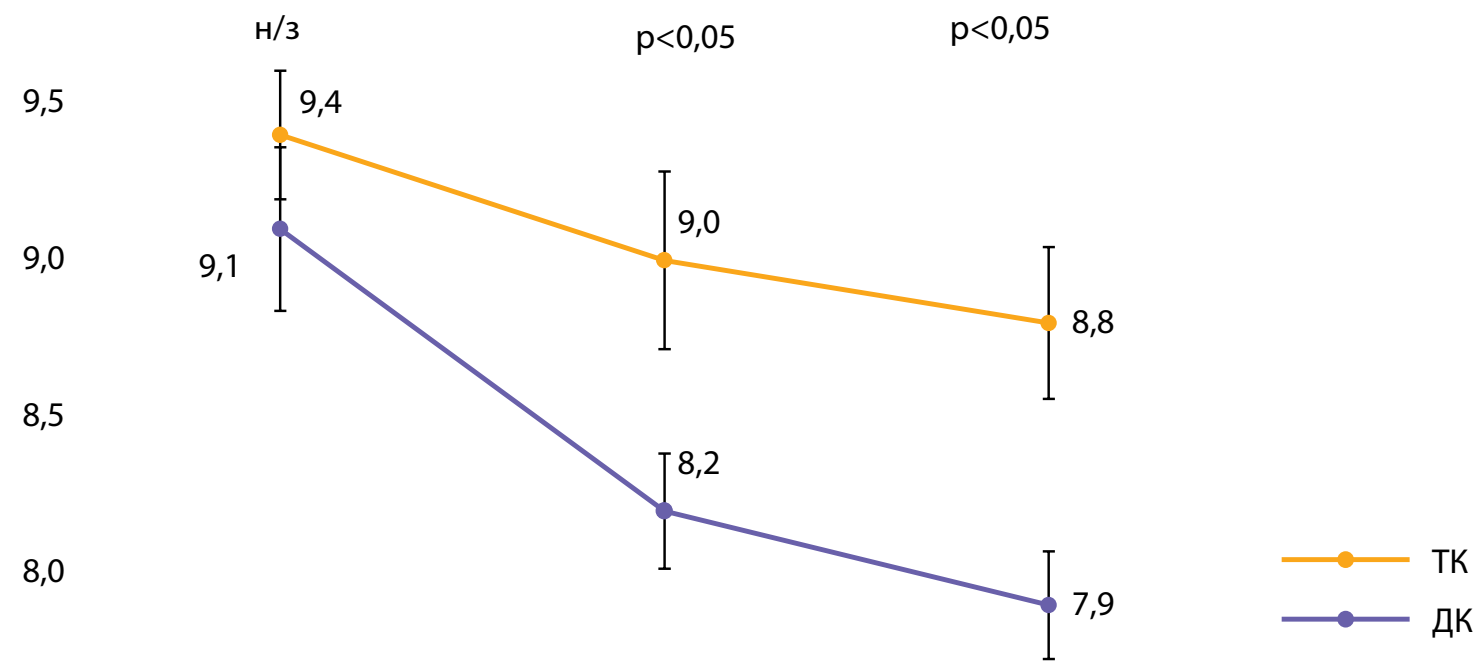

7,5

Исходно 12 недель 24 недели

Рисунок 1. Динамика уровня гликированного гемоглобина $\left(\mathrm{HbA}_{1 с}\right)$ за время исследования в группах традиционного контроля (ТК) и дистанционного консультирования (ДК). Данные представлены в виде $\mathrm{M} \pm \mathrm{SEM}$

Гликированный гемоглобин

Исходно уровень гликированного гемоглобина не различался между группами и составил 9,1 и 9,4\% в группе ДК и ТК соответственно (рис. 1, табл. 2). Через 12 и 24 недели исследования уровень $\mathrm{HbA}_{1 с}$ снизился в обеих группах, достигнув при этом статистически значимых различий между ними.

Снижение уровня $\mathrm{HbA}_{1 c}$ к концу исследования относительно исходного было статистически значимо больше в группе ДК и составило 1,17\% по сравнению с 0,59\% в группе ТК ( $<<0,05$; рис. 2A). Различие в изменении уровня $\mathrm{HbA}_{1 с}$ к концу исследования относительно исходного между группами составило 0,6\%. Доля пациентов, которые достигли целевого уровня $\mathrm{HbA}_{1 c}$ (определяемого как $<7,5 \%)$, была статистически значимо выше в группе ДК (32\%) по сравнению с группой ТК (12,5\%, р<0,05; рис. 2Б).

\section{Вариабельность гликемии, гипо- и гипергликемия}

Исходно вариабельность гликемии, определяемая по уровню SD, частота гипогликемии (гликемия менее 4 ммоль/л) и гипергликемии (гликемия более 10 ммоль/л) не различались между группами (табл. 2). К 12-й неделе исследования отмечены значимо более низкие показатели вариабельности гликемии и частоты гипергликемии, но не гипогликемии. В свою очередь, к концу исследования в группе ДК отмечены статистически значимо более низкие значения показателей SD гликемии, частоты гипо-, гипергликемии. Кроме того, в группе ДК к концу исследования отмечено значимое снижение частоты гипогликемии относительно исходного по сравнению с группой ТК, в которой отмечен незначительный рост уровней данных показателей (рис. 2Г). Также в группе ДК отмечалось более выраженное снижение частоты гипергликемии и вариабельности гликемии к концу исследования относительно исходного уровня, однако динамика не достигла статистически значимого уровня по сравнению с группой ТК (рис. 2B).

\section{Гликемический профиль}

Показатели 8-точечного гликемического профиля исходно значимо не различались между групп, исключая показатели постпрандиальной гликемии после завтрака (табл. 2). Через 12 недель исследования все показатели гликемического профиля, за исключением уровня гликемии перед завтраком, статистически значимо были ниже в группе ДК. К концу исследования все без исключения показатели гликемического профиля были значимо ниже в группе ДК.

\section{Инсулинотерапия}

Проводимая инсулинотерапия, определяемая по уровню среднесуточных доз базального и болюсного инсулина, исходно, в процессе (через 12 недель) и по окончании исследования (через 24 недели) значимо не отличалась между групп, с тенденцией к более высоким дозам инсулина в группе ТК (табл. 2).

\section{Показатели приверженности к лечению}

У пациентов в группе ДК исходно отмечалась более редкая частота замены инфузионной системы по сравнению с пациентами в группе ТК, при этом другие показатели, характеризующие приверженность пациентов к контролю СД, не различались между группами (см. табл. 2). Через 12 недель исследования у пациентов в группе ДК по сравнению с пациентами в группе ТК отмечены значимо более высокие показатели частоты определения гликемии, частоты использования калькулятора болюса и меньшие показатели частоты болюсных введений инсулина «вручную». Однако к концу исследования только показатели частоты самоконтроля глюкозы в крови были 
Таблица 2. Показатели гликемического контроля, гликемического профиля, приверженности лечению, инсулинотерапии, острых осложнений и обращений за медицинской помощью в группах дистанционного консультирования (ДК) и традиционного контроля (ТК).

\begin{tabular}{|c|c|c|c|c|c|c|}
\hline \multirow{2}{*}{ Показатель } & \multicolumn{2}{|c|}{ Исходно } & \multicolumn{2}{|c|}{12 недель } & \multicolumn{2}{|c|}{24 недели } \\
\hline & TK & ДК & TK & Дк & TK & ДК \\
\hline \multicolumn{7}{|c|}{ Гликемический контроль } \\
\hline $\mathrm{HbA}_{1 c^{\prime}} \%$ & $9,4(1,4)$ & $9,1(1,2)$ & $9(1,4)$ & $8,2(1)^{*}$ & $8,7(1,6)$ & $7,9(0,9)^{*}$ \\
\hline Эпизоды гипогликемии, \% & $9,2(15,4)$ & $7,2(10,9)$ & $6,8(12,5)$ & $5,2(4,7)$ & $9,3(15,3)$ & $5(9,4)^{*}$ \\
\hline Эпизоды гипергликемии, \% & $53,1(24,9)$ & $42(23,3)$ & $45,5(22,5)$ & $31,3(20,1)^{*}$ & $43,7(21,3)$ & $29,4(20,9)^{*}$ \\
\hline $\begin{array}{l}\text { Вариабельность гликемии, SD, } \\
\text { ммоль/л }\end{array}$ & $4,7(2)$ & $4,3(1,5)$ & $4,6(1,8)$ & $4(1,4)^{*}$ & $4,5(2,2)$ & $3,8(1,1)^{*}$ \\
\hline \multicolumn{7}{|c|}{ Гликемический профиль 8 точек } \\
\hline Гликемия при пробуждении, ммоль/л & $10,5(2,4)$ & $10,1(2,7)$ & $9,8(2,2)$ & $8,8(2,1)^{*}$ & $9,3(1,8)$ & $8,3(2)^{*}$ \\
\hline Гликемия перед завтраком, ммоль/л & $9,8(2,3)$ & $9,2(2,1)$ & $9,2(2,3)$ & $8,8(2)$ & $8,9(1,8)$ & $8,1(2)^{*}$ \\
\hline $\begin{array}{l}\text { Гликемия через } 120 \text { мин после } \\
\text { завтрака, ммоль/л }\end{array}$ & $10,8(2,8)$ & $9,7(2,9)^{*}$ & $9,8(2,5)$ & $8,5(2,6)^{*}$ & $9,5(2,7)$ & $8,4(2,3)^{*}$ \\
\hline Гликемия перед обедом, ммоль/л & $9,6(2,3)$ & $9,3(2,5)$ & $9,4(2,2)$ & $8,7(2,1)^{*}$ & $9,2(1,8)$ & $8,4(1,8)^{*}$ \\
\hline $\begin{array}{l}\text { Гликемия через } 120 \text { мин после обеда, } \\
\text { ммоль/л }\end{array}$ & $10,3(3,2)$ & $9,8(3,1)$ & $10,2(2,7)$ & $8,6(2,2)^{*}$ & $9,5(2,1)$ & $8,2(2,1)^{*}$ \\
\hline Гликемия перед ужином, ммоль/л & $9,3(2,5)$ & $9,4(2,2)$ & $9,4(2,3)$ & $8,6(1,9)^{*}$ & $9,3(1,6)$ & $8,3(1,7)^{*}$ \\
\hline $\begin{array}{l}\text { Гликемия через } 120 \text { мин после ужина, } \\
\text { ммоль/л }\end{array}$ & $10,6(2,9)$ & $10(2,9)$ & $9,9(2,5)$ & $8,9(2,2)^{*}$ & $9,2(2,2)$ & $8,3(1,8)^{*}$ \\
\hline Гликемия перед сном, ммоль/л & $10,1(2,9)$ & $10,2(2,8)$ & $10,3(2,6)$ & $9,1(2,4)^{*}$ & $9,4(1,9)$ & $8,5(2,1)^{*}$ \\
\hline \multicolumn{7}{|c|}{ Приверженность лечению } \\
\hline $\begin{array}{l}\text { Частота определения гликемии, } \\
\text { кол-во/день }\end{array}$ & $4,8(2)$ & $5,3(2)$ & $5,2(2)$ & $5,8(1,8)^{*}$ & $5,1(2,1)$ & $5,8(2,1)^{*}$ \\
\hline $\begin{array}{l}\text { Частота «ручных» болюсов, } \\
\text { кол-во/день }\end{array}$ & $1,4(1,9)$ & $0,9(1,6)$ & $1,2(1,8)$ & $0,7(1,3)^{*}$ & $1(1,6)$ & $0,7(1,5)$ \\
\hline $\begin{array}{l}\text { Частота использования калькулятора } \\
\text { болюса, кол-во/день }\end{array}$ & $4,8(1,8)$ & $5,2(2,2)$ & $5(1,7)$ & $5,9(1,8)^{*}$ & $5,4(2,3)$ & $5,8(2,1)$ \\
\hline $\begin{array}{l}\text { Частота замены инфузионной } \\
\text { системы, кол-во/2 нед }\end{array}$ & $4,2(2)$ & $3,6(1,4)^{*}$ & $4(1,6)$ & $3,9(1,3)$ & $4(1,9)$ & $4(1,5)$ \\
\hline \multicolumn{7}{|c|}{ Инсулинотерапия } \\
\hline Среднесуточная доза инсулина, ЕД & $43,8(18)$ & $39(17,6)$ & $45,1(17,2)$ & $40,4(17,8)$ & $46,5(17,5)$ & $41,4(17,9)$ \\
\hline $\begin{array}{l}\text { Среднесуточная базальная доза } \\
\text { инсулина, ЕД }\end{array}$ & $19,2(9,1)$ & $16,9(8,1)$ & $20,5(8,6)$ & $17,9(8)$ & $21(8,7)$ & $18,9(8,5)$ \\
\hline $\begin{array}{l}\text { Среднесуточная болюсная доза } \\
\text { инсулина, ЕД }\end{array}$ & $24,5(11,5)$ & $21,9(11,5)$ & $24,7(10,7)$ & $22,7(11,5)$ & $25,5(10,9)$ & $22,5(11)$ \\
\hline \multicolumn{7}{|c|}{ Острые осложнения } \\
\hline $\begin{array}{l}\text { Число эпизодов тяжелой } \\
\text { гипогликемии, кол-во/12 нед }\end{array}$ & $0,1(0,7)$ & $0,2(1,3)$ & $0(0,2)$ & $0(0)^{*}$ & $0(0,1)$ & $0(0)$ \\
\hline $\begin{array}{l}\text { Число эпизодов кетоацидоза, } \\
\text { кол-во/12 нед }\end{array}$ & $0,2(0,7)$ & $0,1(0,3)$ & $0,1(0,2)$ & $0(0,2)$ & $0,1(0,3)$ & $0(0,1)$ \\
\hline
\end{tabular}

\section{Обращение за медицинской помощью}

\begin{tabular}{llcccc}
\hline $\begin{array}{l}\text { Число очных консультаций, } \\
\text { кол-во/12 нед }\end{array}$ & $1,4(1,6)$ & $1,2(1,4)$ & $1,4(1,4)$ & $1(1,1)^{*}$ & $1,3(1,5)$ \\
$\begin{array}{l}\text { Число госпитализаций по } \\
\text { экстренным показаниям, }\end{array}$ & $0,1(0,5)$ & $0,1(0,3)$ & $0(0,2)$ & $0(0,1)$ & $0(0,2)$
\end{tabular}

кол-во/12 нед

Данные представлены в виде M (SD); н/з - статистически не значимые различия; * - уровень статистической значимости (р) менее 0,05 между группами ТК и ДК. 


\section{А. Гликированный гемоглобин}

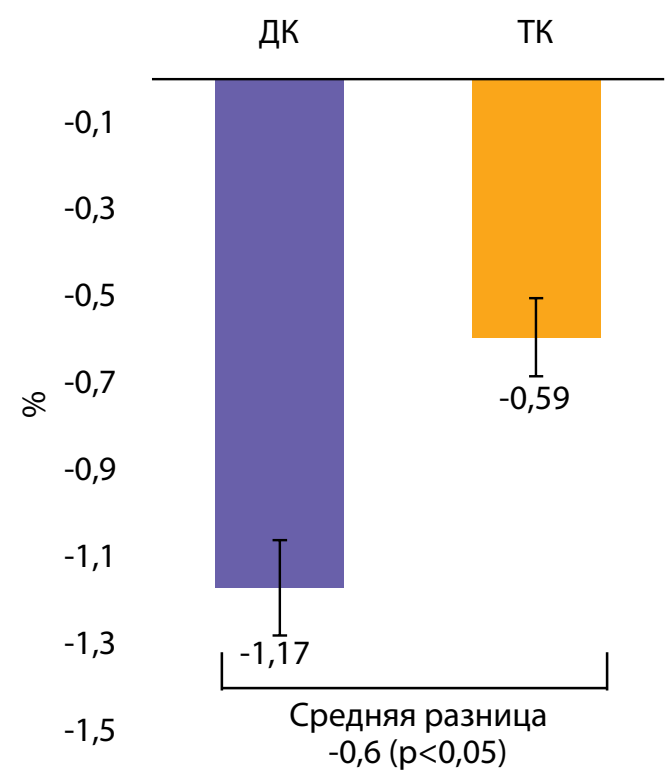

B. Средняя гликемия и SD гликемии

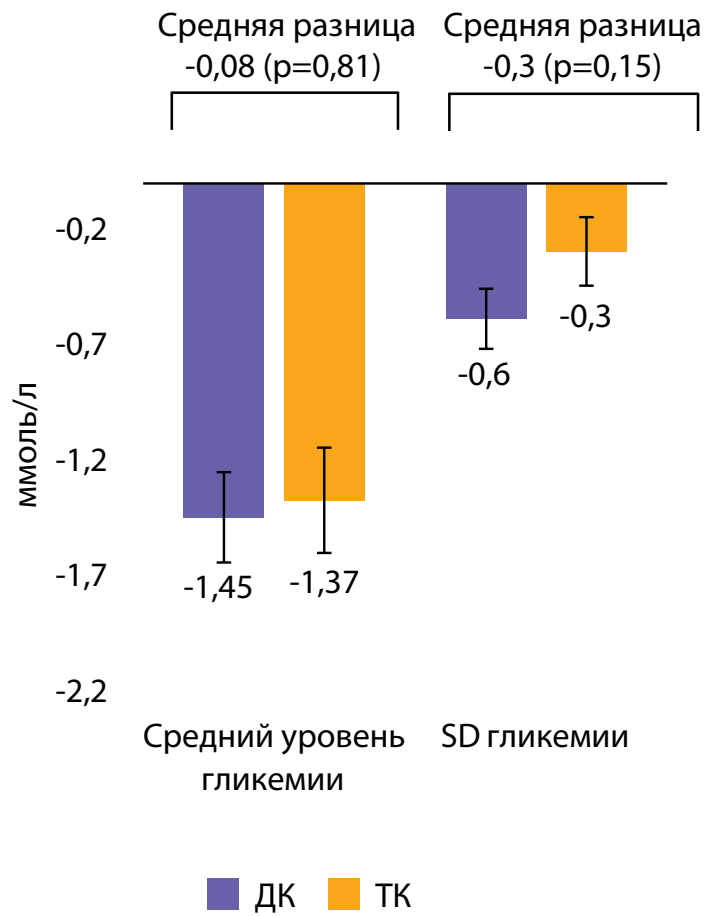

Б. Пациентов с $\mathrm{HbA}_{1 \mathrm{c}}<7,5 \%$

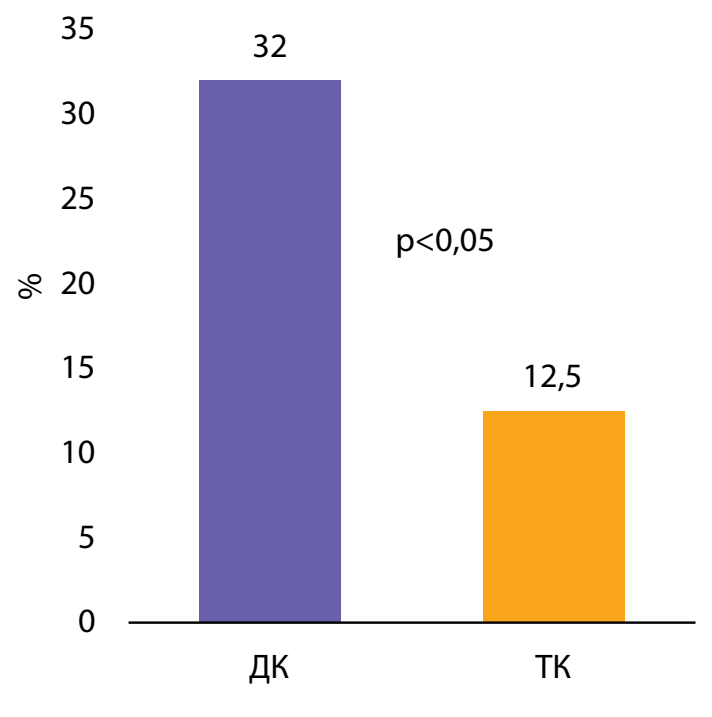

Г. Измерений в диапазоне $<4$ и $>10$ ммоль/л

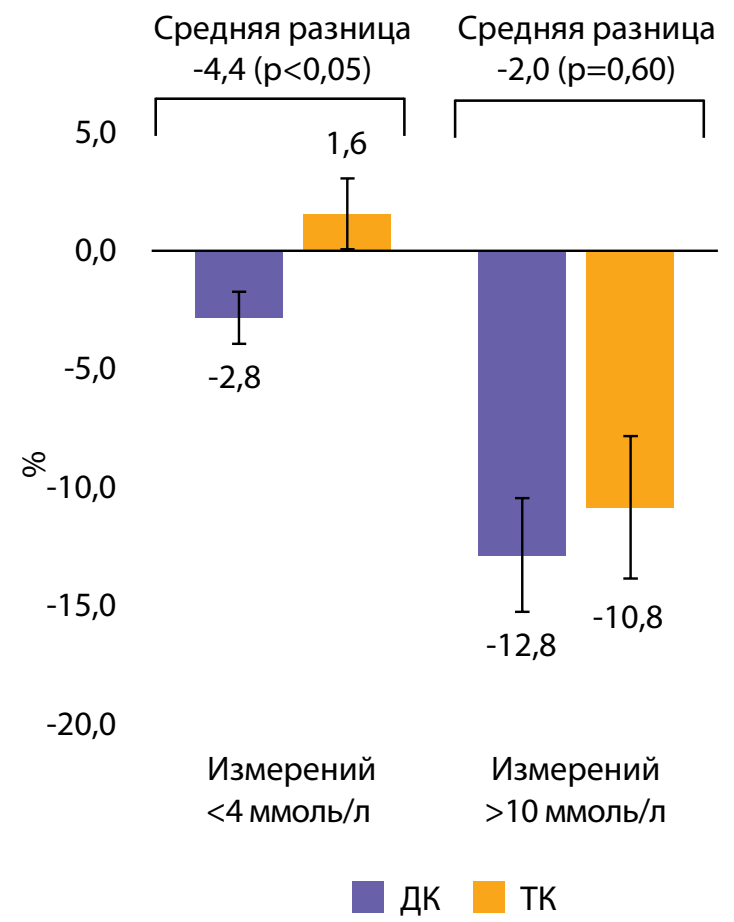

Рисунок 2. Изменение показателей гликемического контроля к концу исследования относительно исходных уровней в группе дистанционного консультирования (ДК) (синие столбцы) и традиционного контроля (ТК) (оранжевые столбцы). Данные представлены в виде М \pm SЕM

значимо выше в группе ДК по сравнению с группой ТК, при этом у пациентов в группе ДК относительно исходного уровня значимо выросла частота замены инфузионной системы по сравнению с пациентами в группе ТК (рис. 3).

\section{Качество жизни}

Исходно отдельные показатели качества жизни различались между группами ДК и ТК (табл. 3). Так, в группе ДК у родителей детей с СД1 был выше один из шести показателей (Лечение-І) качества жизни, а у детей с СД1 были значимо выше три показателя качества жизни (Общий балл, Лечение-І, Лечение-ІІ). В свою очередь, в конце исследования в группе ДК по сравнению с группой ТК все показатели качества жизни были значимо выше у детей с СД1 и три показателя качества жизни - у родителей детей с СД1 (рис. 5). Также к концу исследования отмечена положительная динамика всех показателей в группе ДК по сравнению с группой ТК. Однако статистической значимости достигло изменение к концу исследования по сравнению с исходным уровнем только двух показателей (Общий балл, Диабет) и только у детей с СД1 в группе ДК по сравнению с группой ТК (рис. 3,4$)$. 
Таблица 3. Показатели качества жизни у родителей и детей с сахарным диабетом 1 типа исходно и по окончании исследования. Данные представлены в виде M (SD). Уровень р везде >0,05 при сравнении показателей групп дистанционного консультирования и традиционного контроля.

\begin{tabular}{|c|c|c|c|c|c|c|}
\hline \multirow{2}{*}{ Показатель } & \multicolumn{3}{|c|}{ Исходно } & \multicolumn{3}{|c|}{24 недели } \\
\hline & TK & ДК & $\mathbf{p}$ & TK & ДК & $\mathbf{p}$ \\
\hline \multicolumn{7}{|c|}{ Родители } \\
\hline Общий балл & $60(14,9)$ & $56,5(14,9)$ & 0,145 & $64,5(16,5)$ & $58,6(14)$ & 0,017 \\
\hline Диабет & $54,4(14,1)$ & $53,2(15,7)$ & 0,598 & $59,9(16,5)$ & $55(14,9)$ & 0,055 \\
\hline Лечение-І & $61,7(21,5)$ & $54,9(20,2)$ & 0,043 & $63,7(22,7)$ & $55,5(20,6)$ & 0,019 \\
\hline Лечение-II & $65,4(20)$ & $59,4(22,7)$ & 0,080 & $68,8(20,7)$ & $63(21,9)$ & 0,087 \\
\hline Беспокойство & $61,3(22)$ & $59,3(18,5)$ & 0,546 & $65(22,5)$ & $57,1(17,9)$ & 0,018 \\
\hline Общение & $63,6(25,6)$ & $61,1(22,4)$ & 0,521 & $69(24,3)$ & $63,7(23,5)$ & 0,171 \\
\hline \multicolumn{7}{|c|}{ Дети } \\
\hline Общий балл & $65,2(14,9)$ & $60,2(15,6)$ & 0,045 & $69,7(16,4)$ & $61,1(15,7)$ & 0,001 \\
\hline Диабет & $57,6(15)$ & $57,5(15,8)$ & 0,993 & $64,5(17,2)$ & $57,5(16,5)$ & 0,012 \\
\hline Лечение-І & $71,1(21)$ & $61,7(23,5)$ & 0,011 & $72,4(20,9)$ & $64,5(23)$ & 0,028 \\
\hline Лечение-II & $73,9(19,4)$ & $64,1(22,1)$ & 0,004 & $75,8(19,8)$ & $65,6(21,3)$ & 0,003 \\
\hline Беспокойство & $60,2(25,5)$ & $54,6(19,4)$ & 0,136 & $65,4(22,1)$ & $54,5(20,7)$ & 0,002 \\
\hline Общение & $69,6(21,7)$ & $64,4(23,3)$ & 0,154 & $74,8(21,3)$ & $65,8(25,8)$ & 0,019 \\
\hline
\end{tabular}

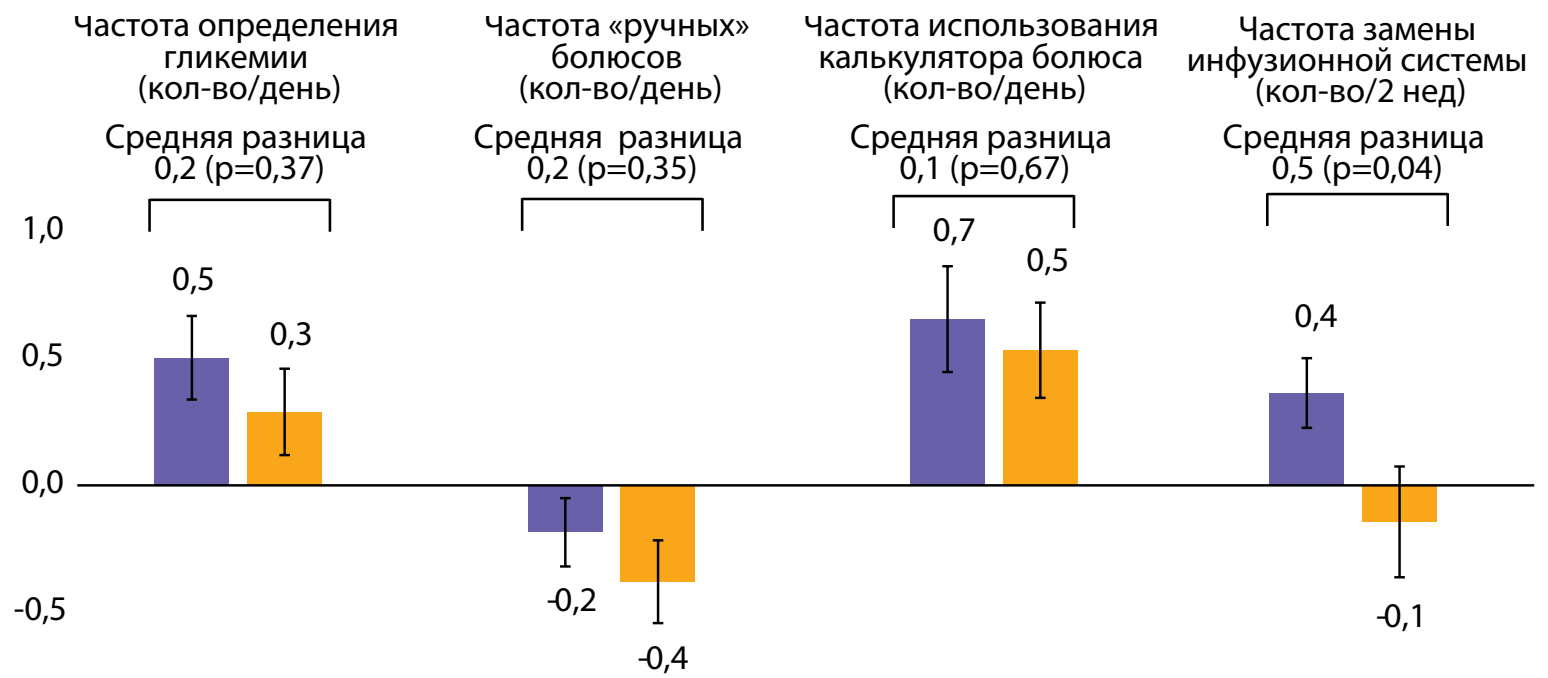

$-1,0$

ДК ТК

Рисунок 3. Изменение показателей приверженности к лечению к концу исследования относительно исходных уровней в группе дистанционного консультирования (ДК) (синие столбцы) и традиционного контроля (ТК) (оранжевые столбцы). Данные представлены в виде M $\pm S E M$

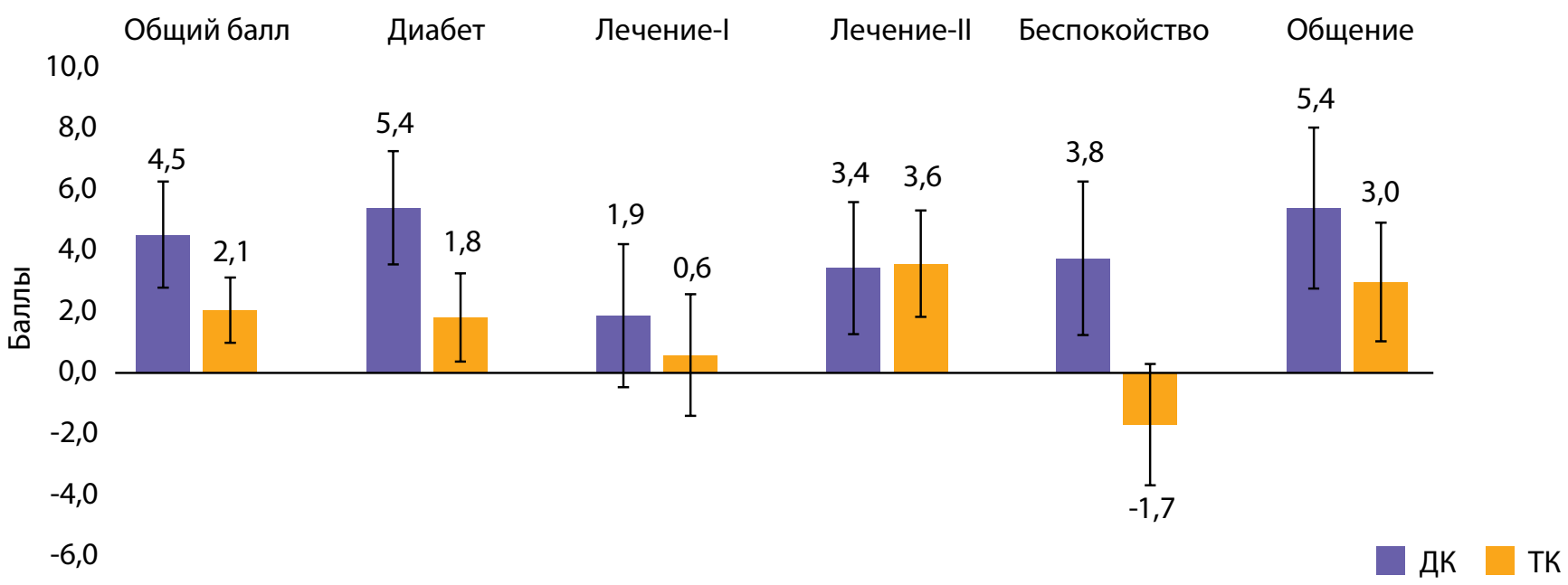

Рисунок 4. Изменение показателей качества жизни у родителей детей с сахарным диабетом 1 типа к концу исследования относительно исходных уровней. Данные представлены в виде среднее \pm SEM. Уровень р везде >0,05 при сравнении показателей групп дистанционного консультирования (ДК) (синие столбцы) и традиционного контроля (ТК) (оранжевые столбцы) 


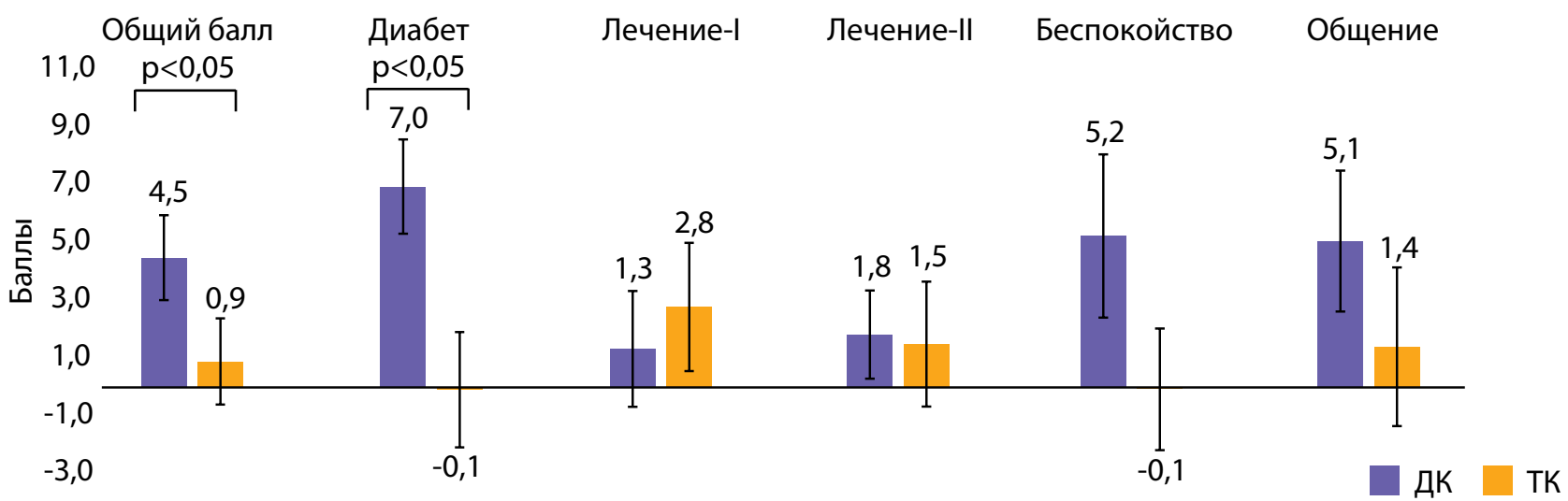

Рисунок 5. Изменение показателей качества жизни у детей с сахарным диабетом 1 типа к концу исследования относительно исходных уровней. Данные представлены в виде среднее \pm SEM.

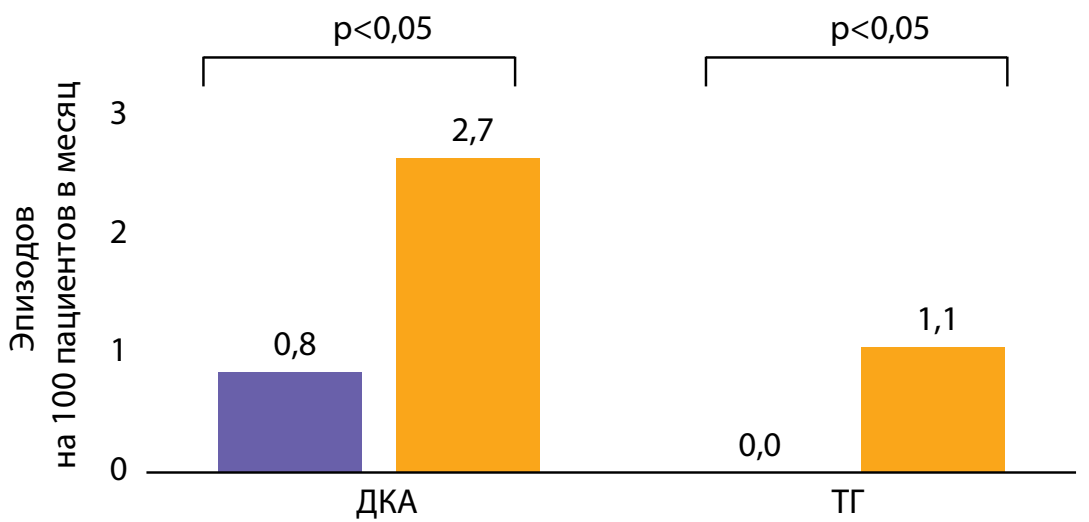

ДК ТК

Рисунок 6. Частота эпизодов тяжелой гипогликемии (ТГ), диабетического кетоацидоза (ДКА) за время исследования в группе дистанционного консультирования (ДК) (синие столбцы) и традиционного контроля (ТК) (оранжевые столбцы). Данные представлены в виде числа случаев на 100 пациентов в месяц

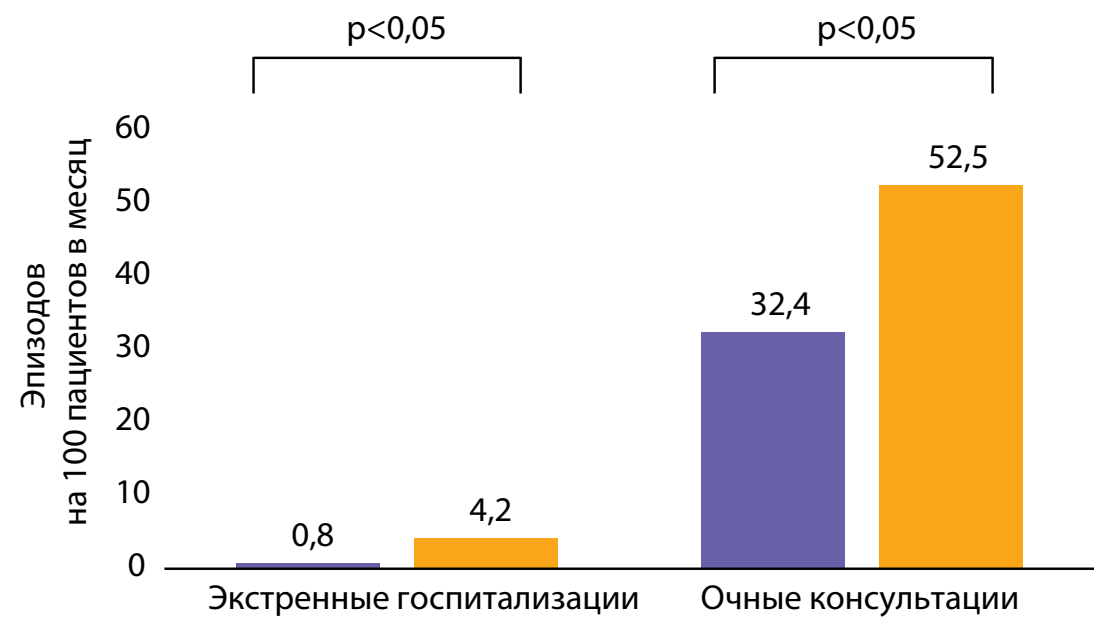

ДК ТК

Рисунок 7. Частота обращений по экстренным/неотложным показаниям и очных консультаций за время исследования в группе дистанционного консультирования (ДК) (синие столбцы) и традиционного контроля (ТК) (оранжевые столбцы), данные представлены в виде числа случаев на 100 пациентов в месяц.

\section{Острые осложнения}

За время исследования частота эпизодов ДКА и тяжелой гипогликемии в группе ДК статистически значимо была ниже у пациентов в группе по сравнению с группой TK (рис. 6).

\section{Обращение за медицинской помощью}

Частота обращений за экстренной и плановой медицинской помощью была значимо ниже у пациентов в группе ДК по сравнению с пациентами в группе ТК (рис. 7).

\section{ОБСУЖДЕНИЕ}

Более низкий уровень $\mathrm{HbA}_{1 с}$ у пациентов с СД1 связан со снижением риска поздних осложнений СД1 [10]. В нашей работе за 24 недели исследования было показано статистически значимое снижение уровня $\mathrm{HbA}_{1 \mathrm{c}}$ в результате дистанционной поддержки детей с СД1 по сравнению с традиционным наблюдением. В ряде исследований также показано снижение уровня $\mathrm{HbA}_{1 с}$ на фоне телемедицинского вмешательства у детей с СД1, и в части из них оно носило статистически 
значимый характер $[11,12]$. Как установлено в нашей работе, снижение уровня $\mathrm{HbA}_{1 c}$ сопровождалось статистически значимым снижением вариабельности гликемии, определяемой по уровню SD, что связано со значимым снижением частоты измерений глюкозы в крови в диапазоне менее 4 ммоль/л и более 10 ммоль/л к концу исследования относительно исходного уровня в группе ДК по сравнению с группой ТК. Таким образом, снижение уровня гликемии было достигнуто не ценой учащения эпизодов гипогликемии, а напротив, сопровождалось снижением эпизодов гипогликемии. Учитывая потенциальный вклад высокой вариабельности гликемии в развитие сосудистых осложнений СД1 [13], показанный в нашем и других исследованиях результат [14] указывает на возможности телемедицины в отношении улучшения дополнительных показателей гликемического контроля.

Более высокая эффективность проводимого лечения может быть связана с повышением уровня знаний пациентов и родителей, лучшей приверженностью лечению и повышением качества жизни на фоне дистанционной поддержки. В нашей работе у пациентов в группе ДК отмечалась тенденция к лучшим показателям приверженности к контролю сахарного диабета (более частый самоконтроль, замена инфузионной системы, использование калькулятора болюса, меньшая частота введения болюсов «вручную»), однако к концу исследования статистически значимо только частота самоконтроля глюкозы была выше в группе ДК. Помимо этого, нами отмечена тенденция к лучшим показателям качества жизни как у родителей, так и детей с СД1 в группе, получавшей дистанционную поддержку, однако статистической значимости достигли только некоторые показатели.

Среднесуточная потребность в инсулине не различалась исходно и в конце исследования между группами. Это может указывать на отсутствие связи полученного эффекта с изменением суточных доз инсулина.

Ни в одном проведенном к настоящему времени исследовании по телемедицине у детей с СД1 не показано учащение острых осложнений СД1, что указывает на безопасность данного вида медицинской помощи [15]. В свою очередь, в нашей работе за время исследования в группе ДК зафиксирована значимо меньшая частота эпизодов острых осложнений СД1, таких как тяжелая гипогликемия и ДКА, по сравнению с группой ТК. Также за время исследования пациенты в группе ДК значимо реже обращались за экстренной и плановой медицинской помощью по сравнению с па- циентами в группе ТК. Указанные обстоятельства могут быть связаны с лучшей доступностью медицинской помощи и лучшим контролем СД1 на фоне дистанционной поддержки и указывают на потенциальные возможности телемедицины в снижении риска острых осложнений СД1, снижении экономических затрат, связанных с перемещением пациента для получения помощи и стоимостью медицинской помощи.

\section{ЗАКЛЮЧЕНИЕ}

У детей с неудовлетворительным гликемическим контролем СД1 дистанционная поддержка оказалась осуществимой и привела к значимому улучшению гликемического контроля $\left(\mathrm{HbA}_{1 c^{\prime}}\right.$ вариабельность гликемии, частота гипогликемии), отдельных показателей приверженности к контролю СД и качества жизни, что сопровождалось меньшей частотой эпизодов ДКА и тяжелой гипогликемии, а также меньшей потребностью в экстренной и плановой медицинской помощи. Накопление доказательств эффективности и безопасности телемедицины при СД должно способствовать внедрению данного подхода в практическое здравоохранение.

\section{ДОПОЛНИТЕЛЬНАЯ ИНФОРМАЦИЯ}

Источник финансирования. Работа выполнена в рамках национальной благотворительной программы помощи детям с эндокринными заболеваниями «Альфа-Эндо» при финансовой поддержке фонда «КАФ». Компания ООО «Медтроник» предоставила для исследования CareLink USB. Компания AO «Байер» предоставила для исследования глюкометры Contour TS и тест-полоски к ним.

Конфликт интересов. Авторы декларируют отсутствие явных и потенциальных конфликтов интересов, связанных с публикацией настоящей статьи.

Участие авторов. Петеркова В.А. - научное руководство, дизайн и планирование исследования; Лаптев Д.Н. - выгрузка данных из регистра, анализ и статистическая обработка полученных данных, написание текста; Емельянов А.О. - ведение регистра помповой инсулинотерапии, выгрузка данных из регистра, написание и редактирование текста; Самойлова Ю.Г. - ведение регистра помповой инсулинотерапии, выгрузка данных из регистра; Храмова Е.Б. - ведение регистра помповой инсулинотерапии, выгрузка данных из регистра; Петряйкина Е.Е. - ведение регистра помповой инсулинотерапии, выгрузка данных из регистра; Рыбкина И.Г. - ведение регистра помповой инсулинотерапии, выгрузка данных из регистра; Филимонова А.Ю. - ведение регистра помповой инсулинотерапии, выгрузка данных из регистра.

\section{СПИСОК ЛИТЕРАТУРЫ | REFERENCES}

1. Петеркова В.А., Кураева Т.Л., Емельянов А.О., и др. Помповая инсулинотерапия сахарного диабета у детей и подростков. Российский консенсус детских эндокринологов // Проблемы эндокринологии. - 2012. - T.58. - №2-2. - C. 2-18. [Dedov II, Peterkova VA, Kuraeva TL, et al. Pompovaya insulinoterapiya sakharnogo diabeta u detei i podrostkov. Rossiiskii konsensus detskikh endokrinologov. Problemy endokrinologii. 2012;58(2-2):2-18 (In Russ.)].

2. Дедов И.И., Петеркова В.А., Кураева Т.Л., Лаптев Д.Н. Инсулиновая помпа (помощь врачу и пациенту для эффективного управления диабетом). — M., 2014. — 128 c. [Dedov II, Peterkova VA, Kuraeva TL, Laptev DN. Insulinovaya pompa (pomoshch'vrachu i patsientu dlya effektivnogo upravleniya diabetom). Moscow; 2014. 128 p. (In Russ.)].

3. Емельянов А.О., Кураева Т.Л., Лаптев Д.Н., Петеркова В.А. Проспективное наблюдение эффективности и безопасности помповой инсулинотерапии у детей и подростков // Сахарный дuaбem. - 2010. — №3. - C. 143-146. [Emel'yanov AO, Kuraeva TL, Laptev DN, Peterkova VA. Prospective study of efficacy and safety of insulin pump therapy in children and adolescents. Diabetes mellitus. 2010;(3):143-146. (In Russ.)]. doi: 10.14341/2072-0351-5503. 
4. Лаптев Д.Н. Преимущество помповой инсулинотерапии и систем мониторирования гликемии в реальном времени в отношении снижения частоты эпизодов гипогликемии у детей и подростков с сахарным диабетом 1-го типа // Проблемы эндокринологии. 2014. - T.60. - №2. - C. 24-30. [Laptev DN. The advantages of insulin pump therapy and real time glucose monitoring systems as the tools for reducing the frequency of hypoglycemic episodes in the children and adolescents with type 1 diabetes mellitus. Problemy endokrinologii. 2014;60(2):24-30. (In Russ.)]. doi: 10.14341/probl201460224-30.

5. Лаптев Д.Н., Филиппов Ю.И., Емельянов А.О., Кураева Т.Л. Оптимизация настроек инсулиновых помп у детей и подростков с сахарным диабетом 1 типа с учетом возрастных особенностей // Сахарный диабеm. - 2013. - №3. - C. 109-115. [Laptev DN, Philippov YI, Emel'yanov AO, Kuraeva TL. Age-adjustment of insulin pump settings in children and adolescents with type 1 diabetes mellitus. Diabetes mellitus. 2013;(3):109-115. (In Russ.)]. doi: 10.14341/2072-0351-98

6. Петеркова В.А., Кураева Т.Л., Емельянов А.О., и др. Помповая инсулинотерапия сахарного диабета у детей и подростков (методическое пособие для педиатров-эндокринологов). M., 2011. —48 c. [Peterkova VA, Kuraeva TL, Emel'yanov $\mathrm{AO}$, et al. Pompovaya insulinoterapiya sakharnogo diabeta u detei i podrostkov (metodicheskoe posobie dlya pediatrovendokrinologov). Moscow; 2011 - 48 p. (In Russ.)]

7. Шестакова М.В., Майоров А.Ю., Филиппов Ю.И., и др. Федеральные клинические рекомендации по помповой инсулинотерапии и непрерывному мониторированию гликемии у больных сахарным диабетом. Проект // Проблемы эндокринологии. - 2015. - Т.61. - №6. - C. 55-78. [Shestakova MV, Mayorov AY, Philippov YI, et al. Russian national guidelines on insulin pump therapy and continuous glucose monitoring for diabetes mellitus patients. Draft. Problemy endokrinologii. 2015;61(6):55-78. (In Russ.)]. doi: 10.14341/probl201561655-78.
8. Дедов И.И., Шестакова М.В., Петеркова В.А., и др. Сахарный диабет у детей и подростков по данным федерального регистра Российской Федерации: динамика основных эпидемиологических характеристик за 2013-2016 гг. // Сахарный duaбem. - 2017. - T.20. — №6. - C. 392-402. [Dedov II,

Shestakova MV, Peterkova VA, et al. Diabetes mellitus in children and adolescents according to the federal diabetes registry in the Russian Federation: dynamics of major epidemiological characteristics for 2013-2016. Diabetes mellitus. 2017;20(6):392-402. (In Russ.)]. doi: 10.14341/DM9460.

9. Izquierdo R, Morin PC, Bratt K, et al. School-Centered telemedicine for children with Type 1 diabetes mellitus. J Pediatr. 2009;155(3):374-379. doi: 10.1016/j.jpeds.2009.03.014.

10. Nathan DM; DCCT/EDIC Research Group. The diabetes control and complications trial/epidemiology of diabetes interventions and complications study at 30 years: overview. Diabetes Care. 2014;37(1):9-16. doi: 10.2337/dc13-2112.

11. Carroll AE, DiMeglio LA, Stein S, Marrero DG. Contracting and monitoring relationships for adolescents with Type 1 diabetes: a pilot study. Diabetes Technol Ther. 2011;13(5):543-549. doi: 10.1089/dia.2010.0181

12. Heidgerken AD, Adkins J, Storch EA, et al. Telehealth intervention for adolescents with type 1 diabetes. J Pediatr. 2006;148(5):707-708. doi: 10.1016/j.jpeds.2006.01.001.

13. Hirsch IB. Glycemic variability and diabetes complications: does it matter? Of course it does! Diabetes Care. 2015;38(8):1610-1614. doi: 10.2337/dc14-2898.

14. Peña NV, Torres M, Cardona JA, Iniesta R. Impact of telemedicine assessment on glycemic variability in children with Type 1 diabetes mellitus. Diabetes Technol Ther. 2013;15(2):136-142. doi: 10.1089/dia.2012.0243

15. Farrell K, Holmes-Walker DJ. Mobile phone support is associated with reduced ketoacidosis in young adults. Diabet Med. 2011;28(8):1001-1004. doi: 10.1111/j.1464-5491.2011.03302.x

Рукопись получена: 22.11.2019. Одобрена к публикации: 24.08.2020. Опубликована online: 20.09.2020.

\section{ИНФОРМАЦИЯ ОБ АВТОРАХ [AUTHORS INFO]}

*Емельянов Андрей Олегович, к.м.н. [Andrey O. Emelyanov, PhD]; адрес: Россия, 117036, г. Москва, ул. Дм. Ульянова, д. 11 [address: 11 Dm Ulyanova str., 117036, Moscow, Russia],

ORCID: https://orcid.org/0000-0002-3742-0231; e-library SPIN: 8110-5540; e-mail: endiab@mail.ru

Лаптев Дмитрий Никитич, д.м.н. [Dmitry N. Laptev, PhD]; ORCID: https://orcid.org/0000-0002-4316-8546; e-library SPIN: 2419-4019; e-mail: laptevdn@ya.ru

Петеркова Валентина Александровна, д.м.н., профессор, академик РАН [Valentina A. Peterkova, Phd, professor, academician of Russian Academy of Medical Sciences]; ORCID: https://orcid.org/0000-0002-5507-4627;

e-library SPIN: 4009-2463; e-mail: peterkovava@hotmail.com

Самойлова Юлия Геннадьевна, д.м.н., профессор [Yulya G. Samoilova, PhD, professor]; ORCID: https://orcid.org/0000-0002-2667-4842; e-library SPIN: 8644-8043; e-mail: samoilova_y@inbox.ru

Храмова Елена Борисовна, д.м.н. [Elena B. Khramova]; ORCID: https://orcid.org/0000-0001-8968-3925; e-library SPIN: 2462-3440; e-mail: doctor.khramova@gmail.com

Петряйкина Елена Ефимовна, д.М.н., професcop [Elena. E. Petryaykina, PhD, professor];

ORCID: https://orcid.org/0000-0002-8520-2378; e-library SPIN: 5997-7464; e-mail: lepet_morozko@mail.ru

Филимонова Алла Юрьевна, к.м.Н., доцент [Alla Y. Filimonova, PhD], ORCID: https://orcid.org/0000-0002-4795-670X, e-library SPIN 4339-3528, e-mail: alla.filimonova.65@mail.ru

Рыбкина Ирина Георгиевна, к.м.н. [Irina G. Rybkina, PhD], ORCID: https://orcid.org/0000-0002-5591-813X,

e-library SPIN 8770-3251, e-mail: ribkinairina@mail.ru

\section{ДЛЯ ЦИТИРОВАНИЯ}

Лаптев Д.Н., Емельянов А.О., Самойлова Ю.Г., Храмова Е.Б., Петряйкина Е.Е., Рыбкина И.Г., Петеркова В.А., Филимонова А.Ю. Дистанционное наблюдение и лечение детей и подростков с сахарным диабетом 1 типа // Проблемы эндокринологии. — 2020. — Т.66. — №4. - С. 50-60. doi: https://doi.org/10.14341/probl12201

\section{FOR CITATION}

Laptev DN, Emelyanov AO, Samoylova YG, Khramova EB, Petriaikina EE, Rybkina IG, Filimonova AY, Peterkova VA. Remote monitoring and treatment of children and adolescents with type 1 diabetes. Problems of Endocrinology. 2020;66(4):50-60. doi: https://doi.org/10.14341/probl12201 